\title{
Numerical Analysis of the Effect of Jet-Fan Operation Condition on Smoke Movement and Visibility in Tunnel Fire
}

\author{
Ha Thien Khieu' ${ }^{1}$, Ji Tae Kim¹, Ki Bea Hong ${ }^{2}$, and Hong Sun Ryou' \\ ${ }^{1}$ Department of Mechanical Engineering, Chung-Ang University \\ 221 Heukseok-dong, Dongjak-gu, Seoul, 156-756, South Korea \\ hathienkhieu@gmail.com; sdd322@naver.com; cfdmec@cau.ac.kr \\ ${ }^{2}$ Department of Automotive Engineering, Korea National University of Transportation \\ 50 Daehak-ro, Chungju, Chungbuk, 27469, South Korea \\ gbhong@ut.ac.kr
}

\begin{abstract}
Every year, many fires occur in the tunnels, with many causalities because of smoke. For this reason, the ventilation system in the tunnel is one of the most critical issues for reducing human causalities. Generally, the jet fan is used for a ventilation system for reducing air pollution in many tunnels. However, when the fire occurs, and jet fan operates, the smoke stratification also happens, it causes a difficulty for people to evacuate. Accordingly, jet fan operating condition is essential to avoid smoke stratification. Besides, the effect of jet-fan on the smoke movement and visibility in the tunnel is also necessarily studied. In this paper, we performed a numerical simulation about the effect of a jet fan on the smoke movement and visibility with variable jet fans speed and different operating condition. The tunnel length is $400 \mathrm{~m}$, there are four jet fans inside, two jet fans are located near the exit position of the tunnel with a diameter of $1.03 \mathrm{~m}$, the distance from it to the exit position and the ceilings are $10 \mathrm{~m}$ and $1.6 \mathrm{~m}$, respectively. Two other jet fans are located at the opposite side, and it is nearby the entrance position with same diameter and distance. The jet fans speeds are from $12.5 \mathrm{~m} / \mathrm{s}, 20 \mathrm{~m} / \mathrm{s}$, and $25 \mathrm{~m} / \mathrm{s}$, respectively. Besides, a different number of jet fans also have been carried out with two jet fans at the exit operate, two jet fans at the entrance operate, and all jet fans operate, respectively. The fire source data was 3.7MW which obtained by our one vehicle sedan fire experiment. Results show that when only two jet fans at the exit are operated, the smoke spreads to both sides, but with other cases, the smoke tends to go to exit direction. Besides, when two jet fans at the entrance are operated, and all jet fans are operated, there is smoke stratification in the tunnel, because in these cases, a large amount of air has been transported into the tunnel by the jet fan operating at the entrance, so the smoke and air are mixed, consequently, the smoke stratification occurs. Furthermore, when jet fan operated at a lower speed, the smoke back-layering length is longer than when operated at higher speed, it also depends on a large amount of air has been transported with high speed. Therefore, it is difficult for evacuation. From the above reasons, jet fans operating condition should be considered for designing a mechanical ventilation system.
\end{abstract}

Keywords: Jet Fan, Heat release rate, Tunnel fire, Smoke movement, Visibility.

\section{Introduction}

The tunnel is an underground passageway with many transportation insides so that investigating the fire in the tunnel is very important for human safety. When a fire occurs, smoke will spread in the tunnel. The component of smoke is $\mathrm{CO}_{2}$ and $\mathrm{CO}$ which is poisoning to humans. Also, as smoke spreads across the tunnel, people cannot see the road to escape from the fire, and they do not know which place they need to go for evacuating, causing many fatalities.

To prevent the smoke from spreading all place in the tunnel, they use the ventilation system to transport smoke from the tunnel to outside. Mechanical ventilation system use exhausted fan or jet-fan to remove smoke in a tunnel fire. Nowadays jet-fan is one of the primary methods for ventilation in the tunnel because jet-fan can quickly move smoke from vehicles and fires to outside. Correspondingly, investigating the jet-fan operation is exceedingly necessary for reducing human causalities.

To determine the interaction between the fire ceiling jet and the flow driven by the jet fan is carried out by João Carlos Viegas (2009) [1]. In that paper, the use of impulse ventilation for smoke control in underground car parks was performed by using Computational Fluid Dynamics (CFD) simulations. Otherwise, the effect of parameters like position and intensity of fire source, restriction of exhaust flow rate and rules for the design jet fans are also studied.

On the other hand, modeling and simulation of a jet fan for controlled air flow in large enclosures are researched by M.G.L.C et al., (2010) [2]. Based on the measurement data and modeling study a relative simple CFD model of the free jet 
fan has been proposed and validated. Guihong Pei and Jie Pan (2014) [3] have investigated the Different Series Modes of Jet Fan in a Longitudinal Tunnel Ventilation System by using numerical study. This method was conducted to research the characteristics of air flow in a tunnel. When most operational fans are located near the tunnel entrance, it is more conductive in increasing the velocity and pressure inside the tunnel. They had already concluded that it is needed to consider various factors when choosing the appropriate series mode in the ventilation system.

Another research has been investigated by Beyer $\mathrm{M}$ et al., (2016) [4] which is an evaluation of jet fan performance in tunnels. Typically, jet fans are used for tunnel ventilation, and due to the lack of space, they are usually installed close to the wall. However, it increased wall shear stress around the jet, so that effectiveness is significantly reduced. Two full-scale experiments of different jet fan applications for existing road tunnels in Austria were performed to analyses installation efficiency. They got the results that the efficiency of the jet fan is depended on tunnel air velocity. The reason for this is that a higher tunnel air velocity always causes an enlargement of the velocity in the flow direction. It induces higher wall friction.

Although there are many kinds of research regarding jet fan operation and efficiency, there is no research relating jet fan in tunnel fires. Accordingly, the numerical study of the effect of the jet fan in the tunnel fires has been carried out by using CFD simulation as a Figure 1. The smoke movement, temperature, velocity, and visibility are also investigated in this paper to clarify the impact of jet fan location and velocity in the tunnel.

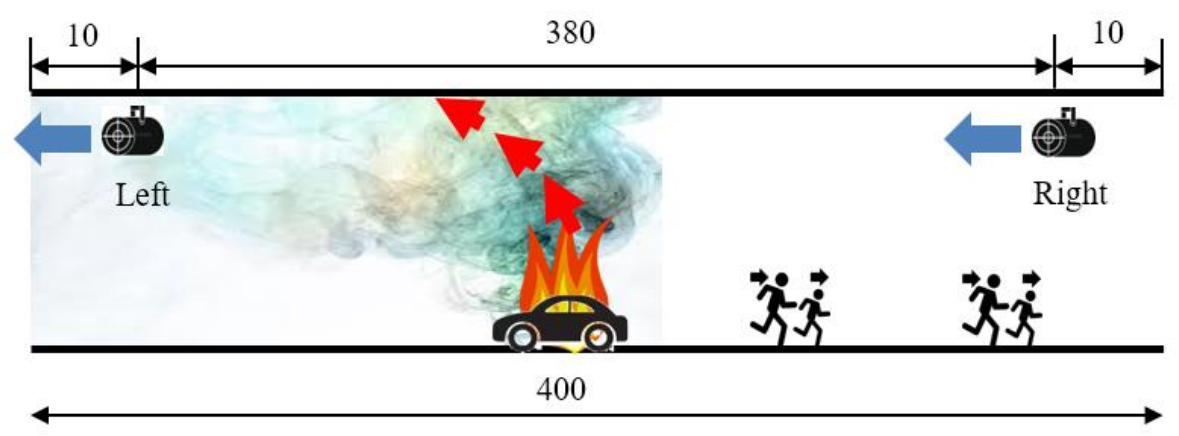

Fig. 1: Numerical analysis objective.

\section{Theoretical analysis}

\subsection{Jet fan}

The jet fan centerline velocity can be calculated as follows

Awbi (2003) [5] and Baturin (1972) [6] provides the equation regarding the centerline velocity and distance that is suitable with the experiment:

$$
\frac{u_{m}}{u_{o}}=\frac{0.48}{\left(a x / d_{o}+0.145\right)}
$$

Where: $\mathrm{u}_{\mathrm{m}}$ is the centerline velocity of the jet fan, $\mathrm{u}_{0}$ is the supply velocity, $\mathrm{x}$ is the distance, a is a constant (from 0.076 to 0.080 for cylindrical tubes), and $\mathrm{d}_{0}$ is the supply diameter of the fan.

With a square duct, Kümmel et al. (2007) [7] provided another equation of jet fan experimental data:

$$
\begin{gathered}
x_{0}=\frac{h}{m} \\
u_{\text {max }}(x)=\frac{x_{0}}{x}
\end{gathered}
$$

In this case: $\mathrm{x}_{0}$ is the length of the potential core, $\mathrm{h}$ is the side length of the square duct, and $\mathrm{m}$ is a constant that is from 0.12 to 0.20 .

From the above equations, we can calculate the jet fan centerline velocity as the figure below with the supply velocity is $25 \mathrm{~m} / \mathrm{s}$, and the side length of the square duct is $0.91 \mathrm{~m}$. 


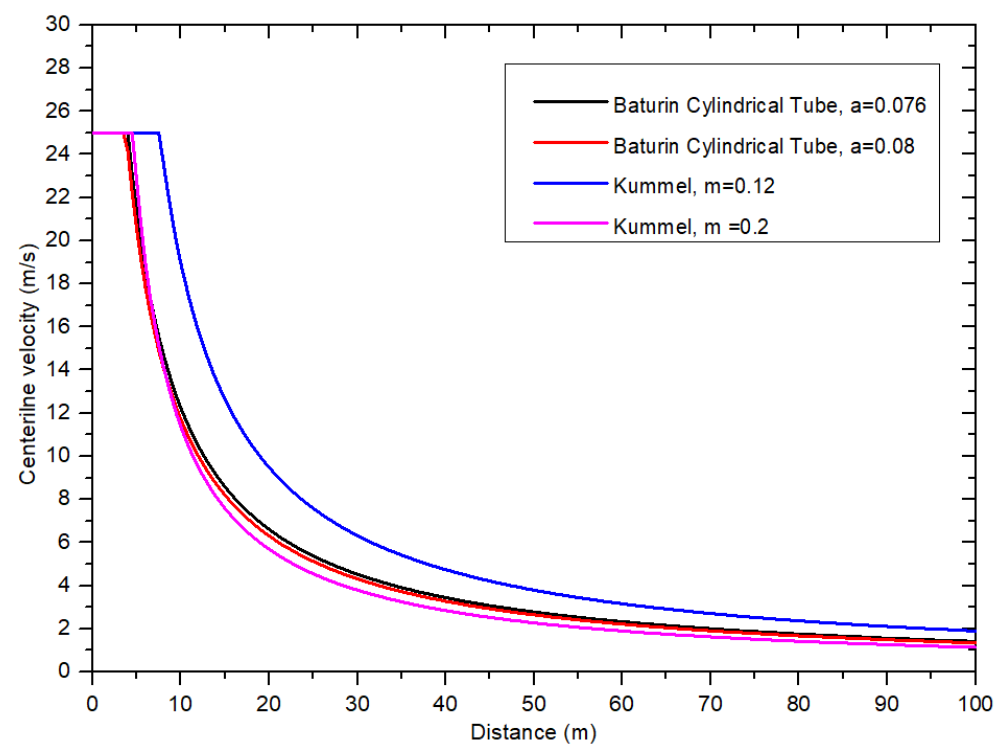

Fig. 2: Jen fan centreline velocity.

\subsection{Heat release rate}

In this study, the heat release rate was got from our experimental study. The maximum heat release rate is $3896 \mathrm{~kW}$ at 1551 second as below figure.

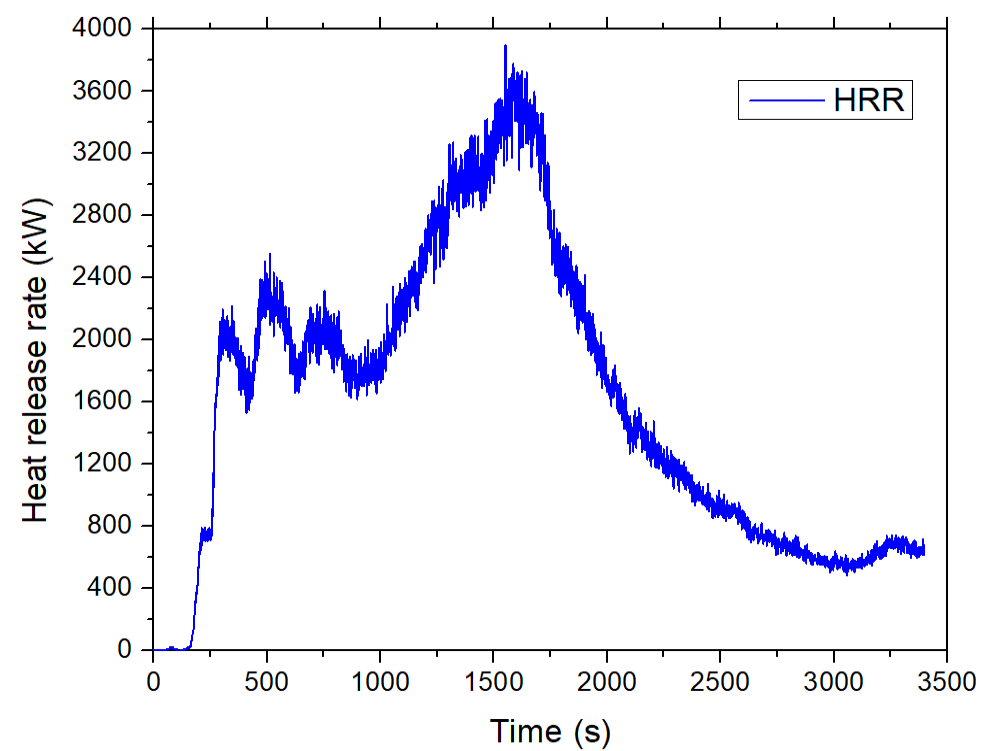

Fig. 3: The heat release rate from an experimental study (one vehicle).

\section{Numerical detail}

\subsection{Geometry and boundary condition}

For this numerical study, we consider the operation condition of the jet fan in tunnel fires. The length of the tunnel is $400 \mathrm{~m}$; there are four jet fans in the tunnel as shown in Figure 4. The computation flow domain was extended over the exit and entrance surface, in order to eliminate the influence of the boundary conditions. Besides, the computational domain was also expanded to the place where the pressure boundary condition was applied. The simulation has been investigated with five cases. Firstly, there are only two jet fans at the exit position (left side) operate. Secondly, there are only two jet fans at 
the entrance position (right side) operate. In case three, all jet fans operate. From case four to case five, there is only two jet fans at the entrance position operate with variable velocity. The value of jet fan velocity and heat release rate as below table.

Table 1: The numerical case study.

\begin{tabular}{|c|c|c|c|c|}
\hline Case & HRR $(\mathbf{k W})$ & Velocity $(\mathbf{m} / \mathbf{s})$ & Left fan & Right fan \\
\hline $\mathbf{1}$ & 3896 & 25 & $\mathrm{O}$ & $\mathrm{X}$ \\
\hline $\mathbf{2}$ & 3896 & 25 & $\mathrm{X}$ & $\mathrm{O}$ \\
\hline $\mathbf{3}$ & 3896 & 25 & $\mathrm{O}$ & $\mathrm{O}$ \\
\hline $\mathbf{4}$ & 3896 & 12.5 & $\mathrm{X}$ & $\mathrm{O}$ \\
\hline $\mathbf{5}$ & 3896 & 20 & $\mathrm{X}$ & $\mathrm{O}$ \\
\hline
\end{tabular}

The length of the tunnel is $400 \mathrm{~m}$, the width and the height of the tunnel are 13.716 and $8.144 \mathrm{~m}$, respectively.

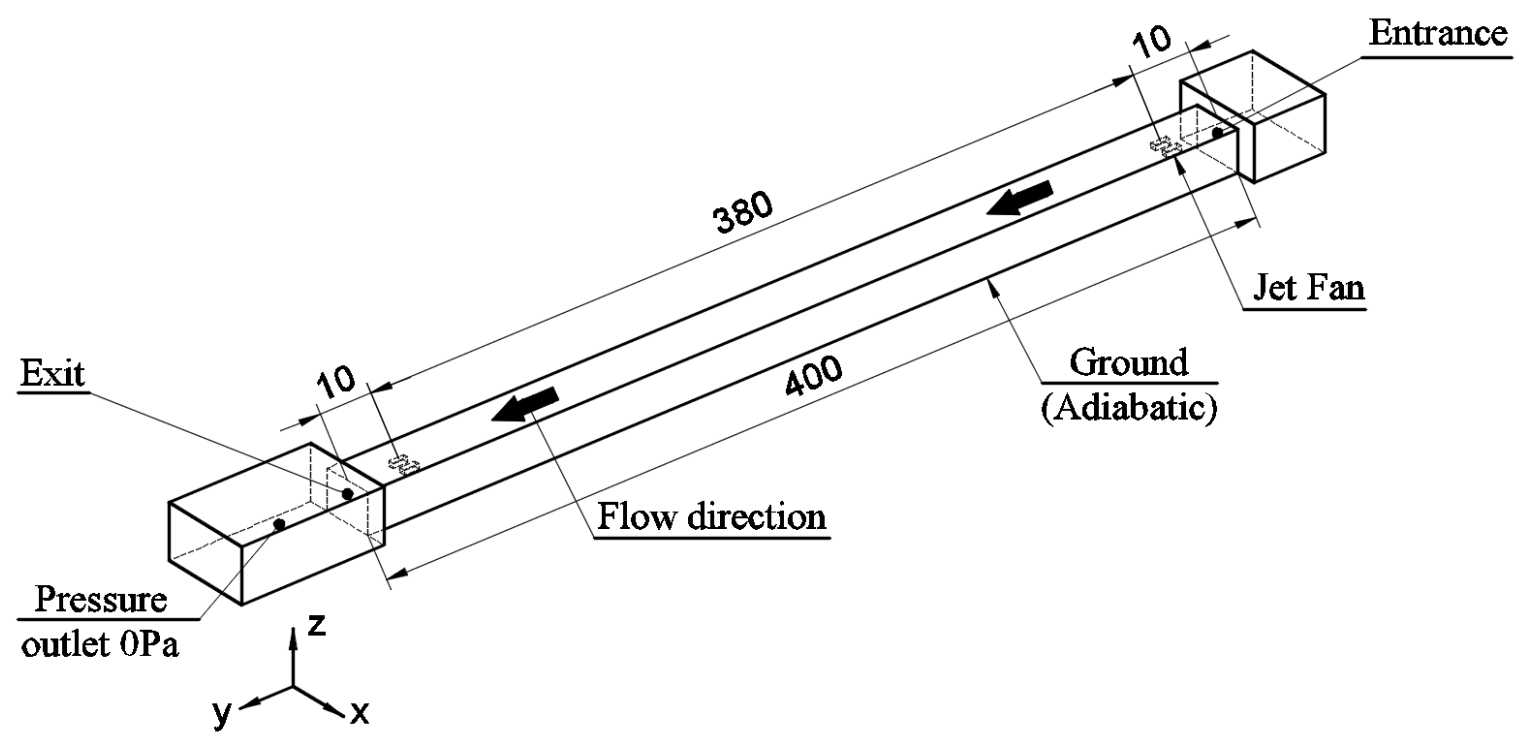

Fig. 4: The geometry of tunnel.

Table 2: The boundary condition.

\begin{tabular}{|c|c|}
\hline Physics & Fluid flow \\
\hline \multirow{3}{*}{ Cell zone } & Gauge pressure $=0 \mathrm{pa}$ \\
& $\mathrm{x}, \mathrm{y}, \mathrm{z}$ velocity $=0 \mathrm{~m} / \mathrm{s}$ \\
& Temperature $: 298.15 \mathrm{~K}$ \\
\hline
\end{tabular}

\subsection{Grid Generation}

National Institute of Standards and Technology already tested show that when the grid size is $1 / 10$ of fire source, the result of the numerical study is agreement with experimental study. In this case, we use grid size $0.1 \mathrm{~m}$ nearby fire source and $0.4 \mathrm{~m}$ far away from the fire source to obtain a good result in the area near the fire source. Besides, the area is far away from the fire source is less critical, so that we use the coarse mesh at that place.

\subsection{Numerical methods}

Fire Dynamics Simulator (FDS) is a computational fluid dynamics software developed by the National Institute of Standards and Technology (NIST) of the United States Department of Commerce, in cooperation with VTT Technical Research Centre of Finland. This computer program numerically solves large eddy simulations of Navier-Stokes equations 
which are suitable for low-speed flow, thermally driven flow, emphasis on smoke and heat transport from fire to describe the progression of the fire.

The governing equations of FDS can be described as follows

Conservation of Mass

$$
\frac{\partial \bar{\rho}}{\partial t}+\frac{\partial \bar{\rho} \tilde{u}_{j}}{\partial x_{j}}=\dot{m}_{b}^{\prime \prime \prime}
$$

Conservation of Momentum (Newton's Second Law)

$$
\bar{\rho}\left(\frac{\partial \tilde{u}_{i}}{\partial t}+\frac{\partial \tilde{u}_{i} \tilde{u}_{j}}{\partial x_{j}}\right)=-\frac{\partial \bar{p}}{\partial x_{i}}+\bar{\rho} g+f_{i}+\frac{\partial \tau_{i j}}{\partial x_{j}}
$$

Conservation of Species

$$
\frac{\partial\left(\bar{\rho} \tilde{Y}_{l}\right)}{\partial t}+\frac{\partial\left(\bar{\rho} \tilde{u}_{j} \tilde{Y}_{l}\right)}{\partial x_{j}}=-\frac{\partial}{\partial x_{j}}\left({\overline{\rho u_{j} Y}}_{l}-\bar{\rho}_{\tilde{u}_{j}} \tilde{Y}_{l}\right)+\frac{\partial}{\partial x_{j}}\left(\overline{\rho D_{l} \frac{\partial Y_{l}}{\partial x_{j}}}\right)+\overline{\dot{w}}_{l}
$$

Conservation of Energy

$$
\frac{\partial(\bar{\rho} \tilde{h})}{\partial t}+\frac{\partial\left(\bar{\rho} \tilde{u}_{j} \tilde{h}\right)}{\partial x_{j}}=\frac{D \bar{p}}{D t}+\frac{\partial}{\partial x_{j}}\left(k \frac{\partial \tilde{T}}{\partial x_{j}}-q_{j}^{r}\right)+\sum_{l} \frac{\partial}{\partial x_{j}}\left(\bar{\rho} D_{l} \tilde{h}_{l} \frac{\partial \tilde{Y}_{l}}{\partial x_{j}}\right)
$$

Regarding simulation of buoyant plumes, a measure of how well the flow field is resolved is given by the nondimensional expression $\mathrm{D}^{*} / \delta \mathrm{x}$, where $\mathrm{D}^{*}$ is a characteristic fire diameter and mesh cell has norminal size $\delta \mathrm{x}$. The quantity $\dot{Q}$ is the total heat release rate of the fire. In this study, the grid size was used by applying the above equation.

$$
D^{*}=\left(\frac{\dot{Q}}{\rho_{\infty} c_{p} T_{\infty} \sqrt{g}}\right)^{\frac{2}{5}}
$$

\section{Results and discussion}

As shown in figure 5, we can see the difference in smoke movement between all cases. In case 1, the smoke propagates both sides, in this case, only two jet fan at the exit position (left side) operate, for this reason, the amount of the fresh air goes into the tunnel is not enough to move smoke from the tunnel to outside. However, in all remain cases, the smoke tends to go toward the exit direction, because, at the entrance position, two jet fans are operating, so that the air can quickly go into the tunnel. In case 2 and case 3, the smoke movement is almost the same. It means that two jet fans operating on the left side are not important. In case 4 , with a smaller velocity $(12.5 \mathrm{~m} / \mathrm{s})$, the smoke back-layering is longer than case $5(20 \mathrm{~m} / \mathrm{s})$. 


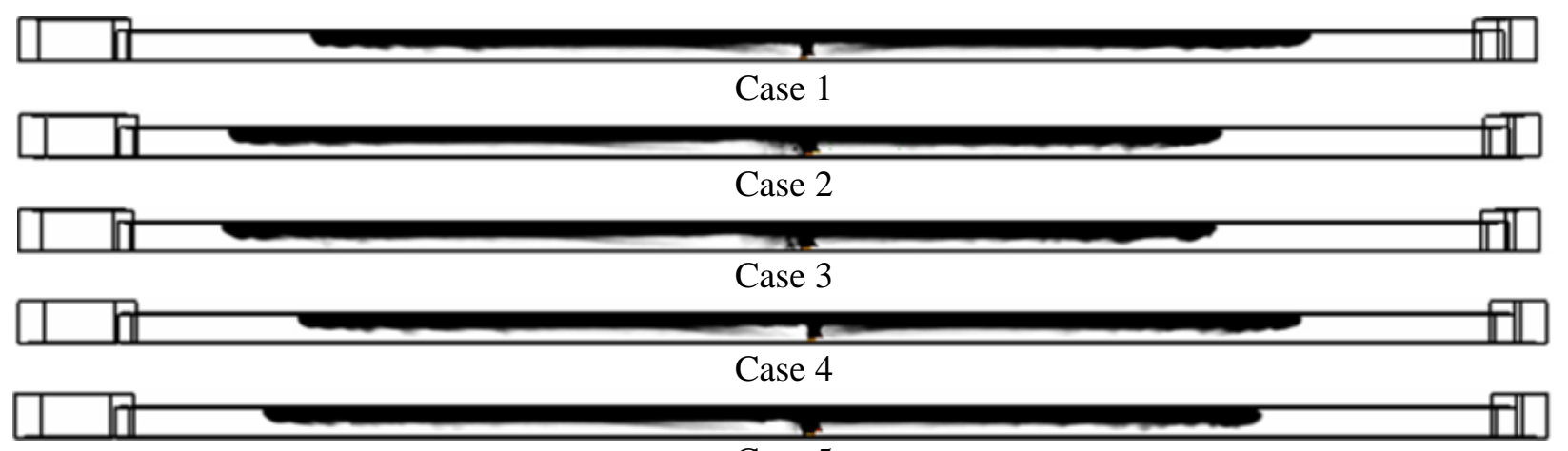

Case 5

Fig. 5: Smoke movement at the time is $1551 \mathrm{~s}$.

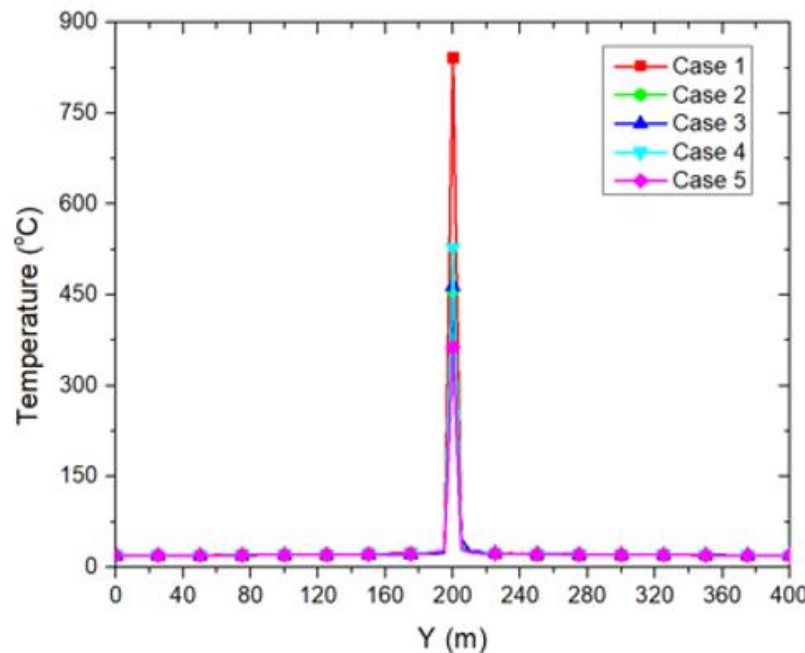

(a)

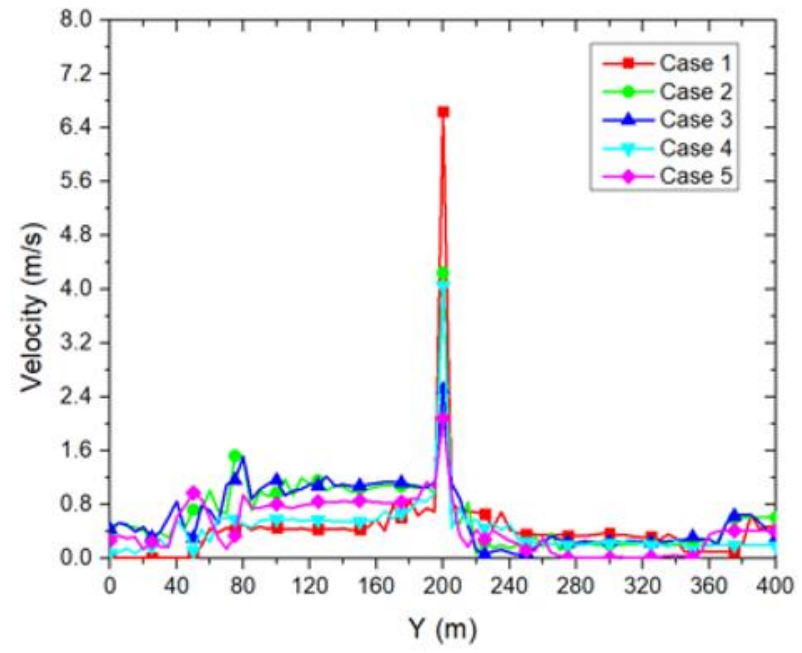

(c)

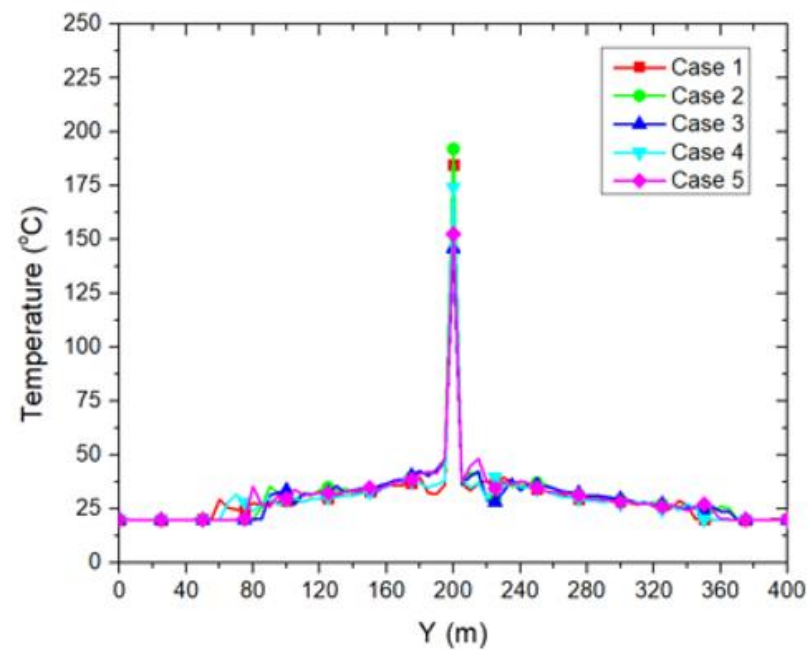

(b)

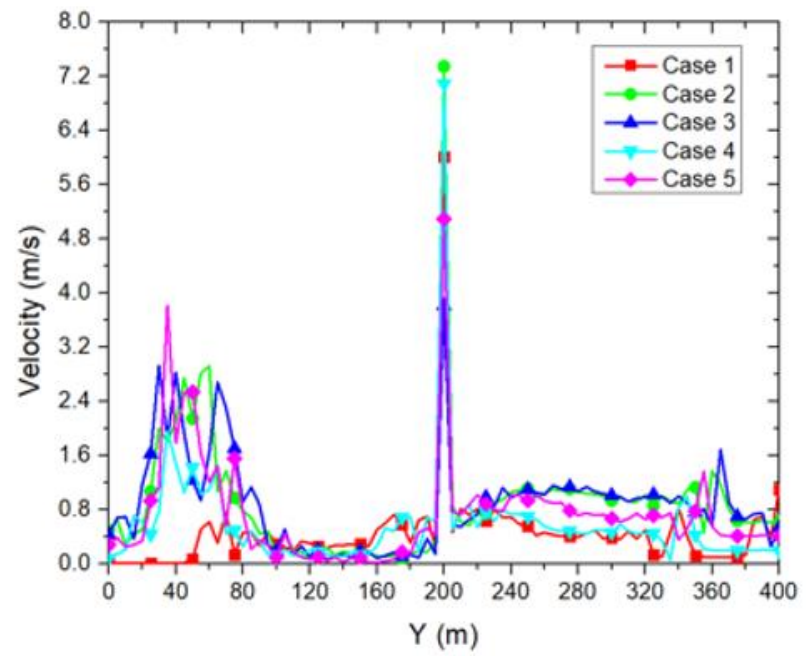

(d)

Fig. 6: Temperature and velocity distribution in the tunnel at the time is $1551 \mathrm{~s}$, (a) Temperature distribution at the height is $1.6 \mathrm{~m}$; (b) Temperature distribution at the height is $5.2 \mathrm{~m}$; (c) Velocity distribution at the height is $1.6 \mathrm{~m}$; (d) Velocity distribution at the height is $5.2 \mathrm{~m}$.

Now, we will consider temperature distribution as the above figure. In figure 6(a), at the height is $1.6 \mathrm{~m}$, all temperatures are the same between 5 cases. At the center of the tunnel, the temperature in case 1 is higher than other cases, because in 
case 1 , only the fan on the left side works, so just a small amount of air enters the tunnel. The fire, in this case, is the most intense. In figure 6(b) the temperature distribution at the height $5.2 \mathrm{~m}$ is entirely different between 5 cases. We will consider the temperature respectively. With case 1 , because only two jet fans on the left side operate so that the smoke spreads to both sides, the temperature nearby the entrance position is higher than other cases. In case 4 , the velocity is lowest $(12.5 \mathrm{~m} / \mathrm{s})$, so that smoke flow rate is quite small, the temperature, in this case, is only lower than case 1, but higher than other cases. After that, there are case 5, case 2 and case 3, respectively. From the above results, not only the position of the jet fan operation but also the velocity of jet fan are significant to design the ventilation system in the tunnel.

The velocity distribution is also shown in figure 6(c) and 6(d). In figure 6(c), when Y is from 0 to 200m, the velocity in case 1 is lowest, because there are no nearby jet fan in this position, but in the center of the tunnel, the fire is the most intense, so that velocity of the smoke is the highest at this point. Similarly, with every position except the center of the tunnel, the higher velocity of the jet fan, the higher the velocity of the smoke.

We continue to investigate the smoke movement in the tunnel after the 1600 s in figure 7 . In case 1 , as we mentioned before, there are only two jet fans on the left side operate; consequently, smoke spreads to both sides. From case 2 to case 5 , the smoke tends to go toward the exit position (left side), but in these cases, we can see the smoke stratification in the tunnel because of the effect of the jet fan. The smoke back-layering in case 3 is shortest because all jet fans in tunnel operate. When the smoke stratification happened, the visibility is decreased, it is difficult for people to evacuate.

As shown in Figure 8, we can see the temperature distribution of the smoke in the tunnel. The smoke temperature distribution is almost the same between 5 cases in figure 8(a), but at the center of the tunnel, the fire in case 1 is also highest intense, the temperature, in this case, is higher than other cases. With figure 8(b), the smoke has spread to the entrance position, so the temperature at places near the entry point in case 1 is higher than the remaining cases. Figure 8(c) and figure $8(\mathrm{~d})$ are the velocity distribution at the height are $1.6 \mathrm{~m}$ and $5.2 \mathrm{~m}$, respectively.

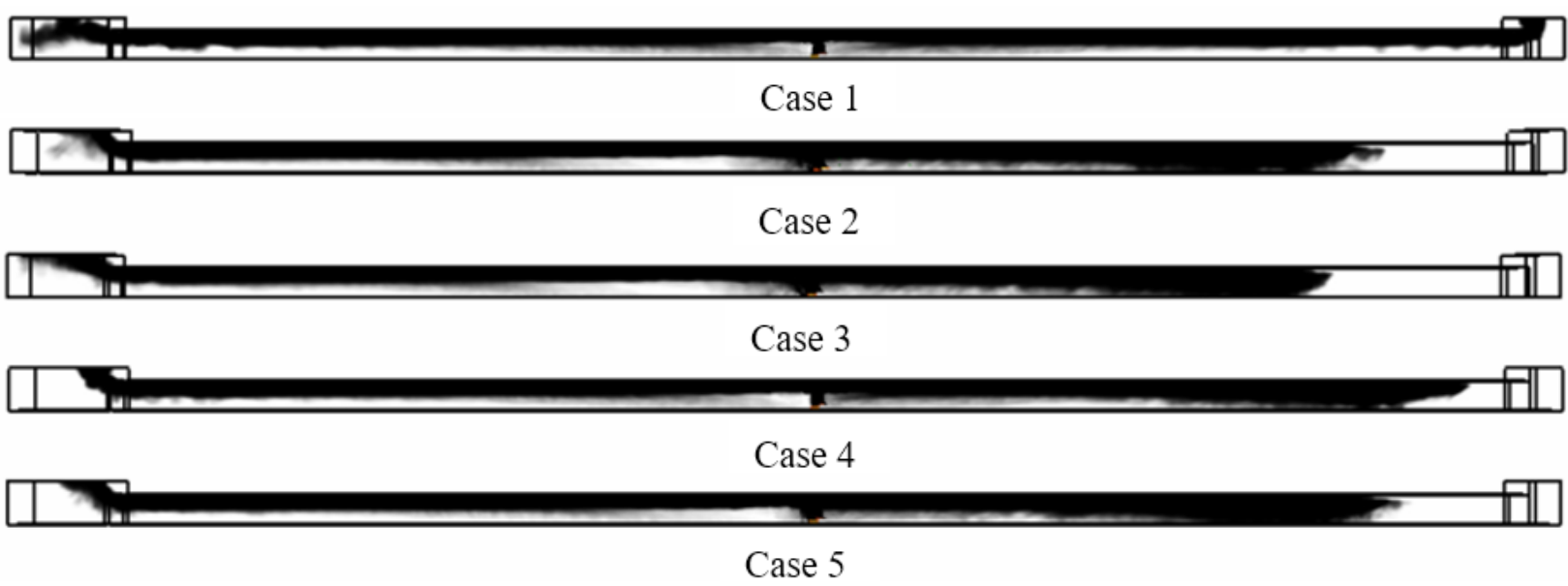

Fig. 7: Smoke movement at the time is the 1600s.

As shown in Figure 9, that is visibility in the tunnel after 1551s. We can realize the difference in visibility among 5 cases. In case 1, because of the spreading of the smoke to both sides, the visibility, in this case, is lower than in other cases. The smoke tends to go in the upper part of the tunnel; it is nearby the ceiling so that the visibility in the upper part of the tunnel is lower than the near floor position. However, after the 1600s as shown in Figure 10, because of the effect of the jet fan on smoke movement, the smoke stratification occurs. All cases except case 1, the smoke spreads to the lower part, so it reduces visibility. In case 3, because of the effect of all jet fans operating, the smoke stratification is highest. 


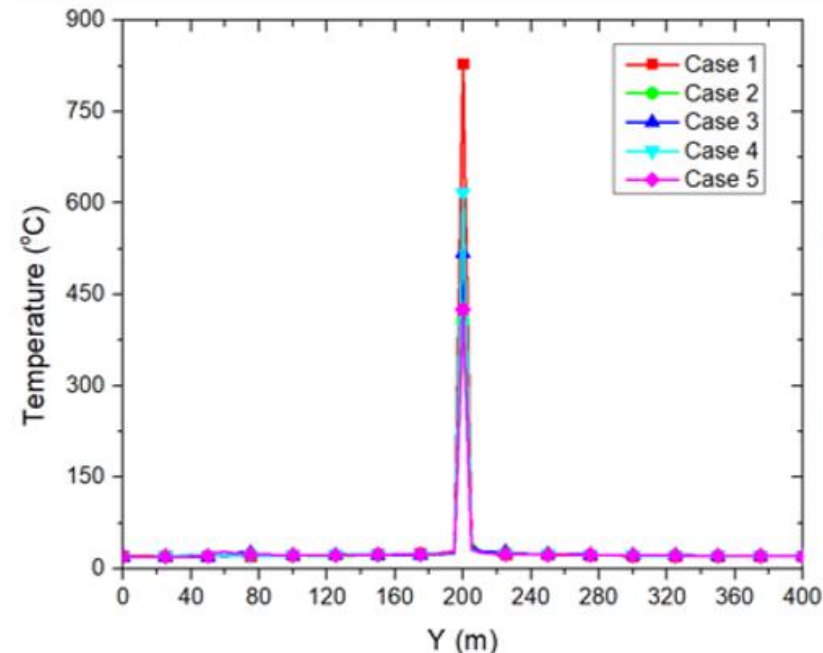

(a)

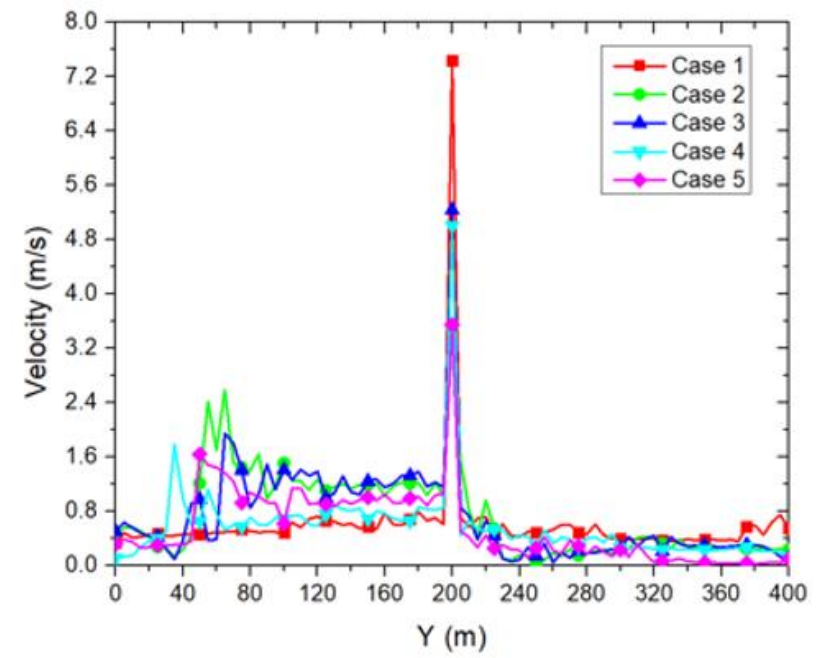

(c)

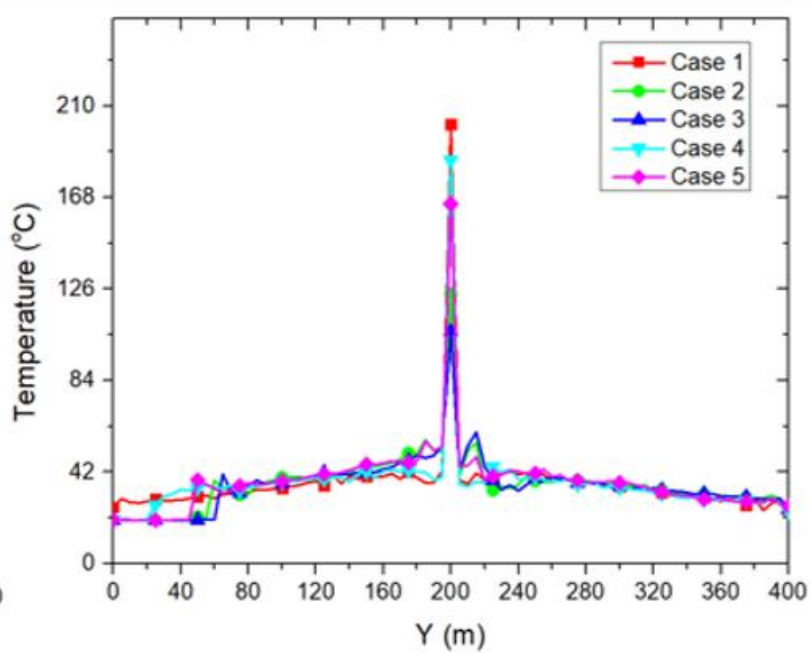

(b)

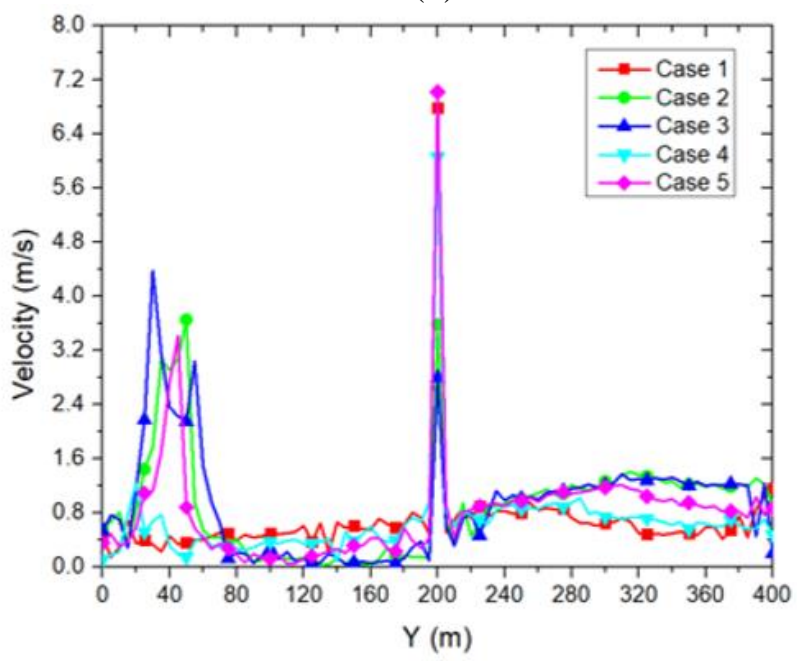

(d)

Fig. 8: Temperature and velocity distribution in the tunnel at the time is the $1600 \mathrm{~s}$, (a) Temperature distribution at the height is $1.6 \mathrm{~m}$; (b) Temperature distribution at the height is $5.2 \mathrm{~m}$; (c) Velocity distribution at the height is $1.6 \mathrm{~m}$; (d) Velocity distribution at the height is $5.2 \mathrm{~m}$.

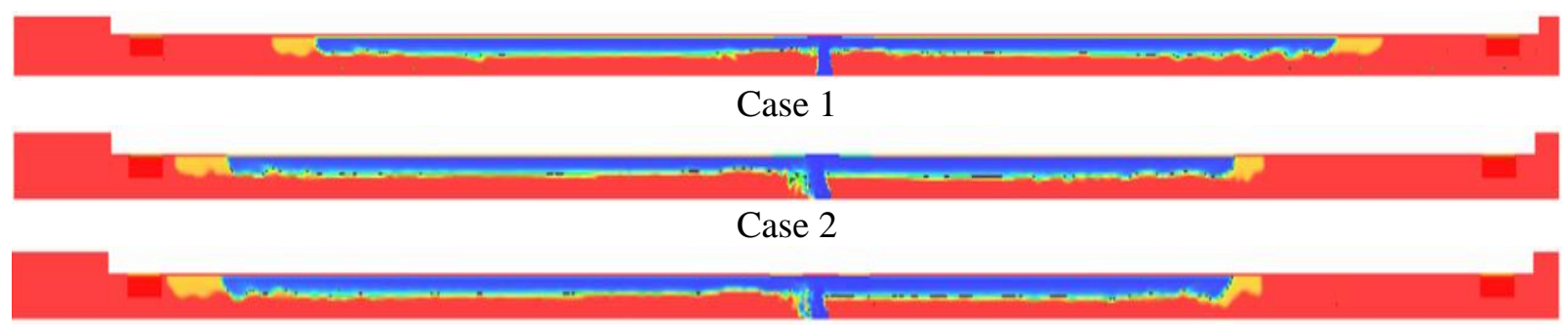

\section{Case 3}

Case 4

Case 5

Fig. 9: Visibility at $\mathrm{x}=0$, time is the $1551 \mathrm{~s}$. 


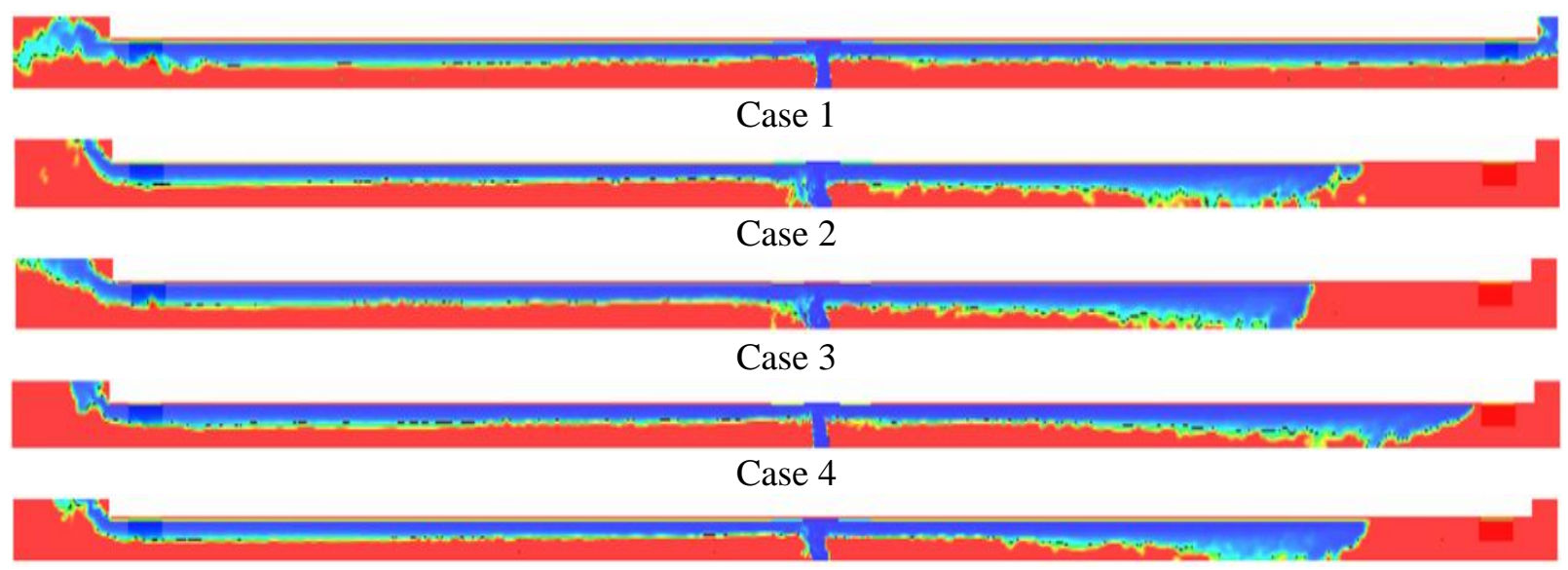

Case 5

Fig. 10: Visibility at $\mathrm{x}=0$, time is the 1600 s.

\section{Conclusion}

In this study, we performed the jet fan operation by numerical methods with variable jet fans speed and different positions of jet fans operating. The effect of jet fan operation condition on smoke movement and visibility in tunnel fire are drawn as follows:

When only two jet fans at the exit position are operated, the smoke spreads to both sides, but with other cases, the smoke tends to go to the exit direction. Because in this case, jet fans at the entrance position are operated, therefore a large amount of air smoothly goes into the tunnel. The result shows that the smoke has to spread to the exit direction because of the effect of the jet fan.

When only two jet fans at the entrance position are operated, and all jet fans are operated, the smoke stratification occurs, because in these cases, the air flow rate go inside is higher than case 1, so that the smoke and air are mixed, from this reason, the smoke stratification occurs, it is difficult for evacuation.

After changing the speed of the jet fan, the lower speed it is, the longer the smoke back-layering it is. It also depends on a small amount of air has been transported at low speed.

Visibility also varies between cases. As shown in case 1, the smoke goes to both sides, so that the visibility on the right side is lower than in other cases.

For these reasons, it is necessary to consider the jet fan operation condition to design the mechanical ventilation system in the tunnel.

\section{Acknowledgment}

This research was supported by the Korea National University of Transportation in 2018 and Alllitelife Company.

\section{References}

[1] João Carlos Viegas, "The use of impulse ventilation for smoke control in underground car parks," Tunneling and Underground Space Technology, vol. 25 no. 1, pp. 42-53, 2010.

[2] B. J. M. vd Giesen, et al. "Modelling and simulation of a jet fan for controlled air flow in large enclosures," Environmental modeling \& software, vol. 26, no. 2, pp. 191-200, 2011.

[3] Guihong Pei, and Jie Pan. "Numerical study on different series modes of the jet fan in a longitudinal tunnel ventilation system," Mathematical Problems in Engineering, 2014.

[4] Michael Beyer, et al. "Evaluation of Jet Fan Performance in Tunnels," 8th International Conference Tunnel Safety and Ventilation, 2016.

[5] Hazim B. Awbi, Ventilation of Buildings, Second edition, Spon Press, 2003.

[6] V. V. Baturin, Fundamentals of Industrial Ventilation, Pergamon, Oxford, 1972.

[7] Kümmel, Technische strömungsmechanik, Technical report, B.G. Teubner, 3. Auflage, 2007. 25 
[8] Joon-Hyung Kim, et al. "High-efficiency design of a tunnel ventilation jet fan through numerical optimization techniques," Journal of mechanical science and technology, vol. 26, no. 6, pp. 1793-1800, 2012.

[9] Milan B. Šekularac, "Experimental determination of tunnel ventilation axial ducted fan performance," Thermal Science, vol. 20, no. 1, pp. 209-221, 2016.

[10] W. J. A Dahm, and P. E. Dimotakis, "Measurements of entrainment and mixing in turbulent jets," AIAA Journal, vol. 25, no. 9, pp. 1216-1223, 1987.

[11] Sung Ryong Lee and Hong Sun Ryou. "A numerical study on smoke movement in longitudinal ventilation tunnel fires for different aspect ratio," Building and Environment, vol. 41, no. 6, pp. 719-725, 2006.

[12] L. Y. Cooper, M. Harkleroad, J. Quintiere and W. Rinkinen, "An experimental study of upper hot layer stratification in full-scale multiroom fire scenarios,” J. Heat Transfer, vol. 104, pp. 741-749, 1982.

[13] M. Janssens and H. C. Tran, "Data reduction of room tests for zone model validation," J. Fire Sci., vol. 10, pp. 528$555,1992$. 